\title{
TEXTPRODUKTION KOGNITIVE TEXTMODELLE
}

\section{EINLEITUNG}

Im Rahmen des Forschungsansatzes "Kognitive Wissenschaft" hat sich in den letzten zwei Jahrzehnten die Kognitive Linguistik stürmisch entwickelt und durch ihre Arbeiten neue Akzente in der gesamten sprachwissenschaftlichen Forschung gesetzt. Im Mittelpunkt des Interesses der Kognitiven Linguistik steht die Erforschung der Interaktion zwischen der mentalen Repräsentation und der Verarbeitung sprachlichen Wissens.

Als ein konkretes Anwendungsfeld dieses neuen Ansatzes hat sich schon früh die Forschung zum "Verarbeiten und Verstehen von Texten" und zum "Lernen mit Texten" entwickelt. ${ }^{1}$ In diesem Forschungsansatz verbinden sich Gesichtspunkte und Impulse aus sehr verschiedenen Disziplinen wie der kognitiven Psychologie, der Informationsverarbeitungstheorie, der Linguistik, insbesondere der Textlinguistik, der ebenfalls neu aufkommenden Textwissenschaft, der Psycholinguistik und der Computerwissenschaft (vgl. Norman 1981, Shechter \& Toglia 1985).

Anders verlief die Entwicklung in einem anderen Forschungsfeld von Kognitiver Wissenschaft, dem "Textproduzieren". Erst seit Mitte der 80er Jahre kann man ein wachsendes Interesse auch an den produktiven Verwendungsweisen von Sprache beobachten.

An die Textproduktion kann man mit unterschiedlichen Interessen und Fragen herangehen: Zum einen kann man sich für das Produkt interessieren und z.B. fragen, ob der Text unter Gesichtspunkten der Grammatik korrekt ist oder ob der Text kohärent, themabezogen ist. Dies sind linguistische und texttheoretische Fragen zum Textproduzieren. Zum anderen kann man sich für den Prozeß interessieren und z.B. fragen, welches Wissen und welche Handlungen ein Textproduzent einsetzen muß, um sein eigenes Vorgehen bei der Textproduktion zu kontrollieren und zu steuern, und welche Rückwirkungen der Textproduktionsprozeß auf das Wissen des Textproduzenten hat. In diesem Fall handelt es sich um eine prozeßorientierte Betrachtungsweise von

1 Textverstehen als auch Lernen mit Texten sind in den USA mittlerweile wohletablierte Forschungsthemen; vgl. z.B. Kintsch \& van Dijk 1978. 
Textproduzieren, d.h. um eine Sicht des Textproduzierens, die die Kognition in den Mittelpunkt des Forschungsinteresses setzt. Die zentralen Fragen richten sich dabei auf die repräsentationalen und prozeduralen Aspekte der Sprachverarbeitung: Welche Planungseinheiten liegen der Sprachproduktion zugrunde? Wie werden die Einheiten so unterschiedlicher Kenntnissysteme wie Semantik und Phonologie abgerufen? Laufen die Prozesse unabhängig voneinander ab oder interagieren die Prozeßkomponenten miteinander? Inwieweit determiniert der Kontext den Verbalisierungsprozeß?

Eine Textproduktionstheorie sollte nun beschreiben und erklären, wie mentale Repräsentation in (schriftliche oder mündliche) Sprachstrukturen übersetzt wird. Die Untersuchung bewußter und unbewußter Planungsprozesse bei der Textproduktion hat zwar eine lange Tradition, jedoch ergibt sich hier - wie bei der Erforschung aller mentalen Phänomene - das Problem, daß die Prozeßebenen der Beobachtung nicht direkt zugänglich sind. Sie müssen mittelbar über externe Parameter erschlossen und mittels theoretischer Modelle rekonstruiert werden. Ziel der Textproduktionsforschung ist es, die involvierten Planungs- und Aktivierungsvorgänge im Rahmen eines umfassenden Prozeßmodells (das Modell muß u.a. auch berücksichtigen, daß es sich dabei um in der Zeit ablaufende und von der Gedächtniskapazität eingeschränkte Vorgänge handelt) zu explizieren.

Im folgenden werden Einblicke in grundlegende kognitive Prozesse der Planung und Produktion von Texten gegeben (Abschnitt 2), dann werden die in der kognitiv orientierten Textproduktionsforschung entwickelten Annahmen und Modelle dargestellt (Abschnitt 3), wobei an manchen Punkten weitergehende Überlegungen angestellt werden. Schließlich werden auch wesentliche empirische Methoden referiert (Abschnitt 4), die u.a. auch der Überprïfung und Falsifizierung bestimmter Annahmen dienen. Im letzten Abschnitt (5) werden einige präzisierende Textproduktionsprozeßannahmen getroffen.

\section{ALLGEMEINE ÜBERLEGUNGEN ZUR KOGNITION BEIM TEXTPRODUZIEREN}

Eine Vorbedingung von Textproduzieren ist Wissen. Wissen ist die Menge aller Informationen, die ein Mensch intern gespeichert hat. Wissen ist nicht nur eine statische Ansammlung von Erfahrungsinhalten, sondern auch die Fähigkeit, auf diesen Inhalten zu operieren. Die allgemeine kognitive Kompetenz des Menschen umfaßt somit deklaratives, prozedurales und episodisches Wissen.

Deklaratives Wissen ist Wissen über die Welt. Es wird in Form von Netzwerken, Propositionenlisten, Eigenschaftsrelationen, Teil-Ganzes-Beziehungen usw. beschrieben. Prozedurales Wissen, auch Handlungswissen genannt, dient dem Umgang mit der Welt. Damit sind in erster Linie diejenigen außersprachlichen Wissensbestände 
angesprochen, die es uns erlauben, bestimmte Ereignisse als bestimmte Handlungen zu deuten und selbst Handlungen durchzuführen. Es geht also um ein prozessual orientiertes Wissen, das uns z.B. auch befähigt, in einer bestimmten Kommunikationssituation bestimmte Handlungen unserer Kommunikationspartner zu erwarten. Wir wissen außerdem, welche Handlungen in bestimmten Situationen normalerweise aufeinander folgen, d.h. unser Wissen umfaßt ganze Handlungskomplexe, die gewöhnlich auch kulturell geprägt sind (vgl. Watzlawick et al. 1974). Prozedurales Wissen wird oft in Wenn-Dann-Beziehungen beschrieben. Episodisches Wissen ist Wissen über Erlebtes, d.h. biographisches Wissen.

Deklaratives Wissen beim Textproduzieren ist das Wissen von Textproduzenten über die Aufgabe Textproduzieren. Hierzu gehört insbesondere der kommunikative Rahmen beim Textproduzieren, also Wissen über Zweck und Funktion von Textproduzieren, über Thema, Zeit, Leser.

Prozedurales Wissen beim Textproduzieren ist das Wissen des Textproduzenten über Strategien, d.h. über seine Handlungsmöglichkeiten zur Textproduktion wie z.B. Planung (Gliederung, Skizze usw.), Generierung (aus dem Langzeitgedächtnis oder aus unterschiedlichen externen Informationen) oder Formulierung.

Es ist sinnvoll, daß beim Textproduzieren zwischen einer Bedingungsebene und einer Handlungsebene unterschieden wird. Der Textproduzent selbst ist beiden Ebenen zuzuordnen.

\subsection{Die Handlungsebene}

Das Textproduzieren ist als Handlungsverlauf zu bestimmen (vgl. Rehbein 1977, Ehlich 1980, Ehlich \& Rehbein 1979). Eine weitere Eigenschaft von Textproduzieren ist, daß die Handlungen sich auf mehrere Ebenen beziehen, so wie sich auch ein Text aus mehreren Ebenen zusammensetzt. Die Forscher nehmen ähnliche Aufteilungen von Handlungen vor. Auf einer ersten Ebene wird das Textproduzieren in zwei Typen von Handlungen unterteilt, die sich generell unterscheiden: Planung und Ausführung.

\subsubsection{Planung}

Planen als ein stetiger und dynamischer Prozeß bedeutet, Entscheidungen zwischen Alternativen zu fällen. Beim Textproduzieren sind zwei Arten von Planung zu unterscheiden: die inhaltliche Planung und die Planung der Vorgehensweise (s. z.B. Flower \& Hayes 1981b).

\subsubsection{Inhaltliche Planung}

Bei der inhaltlichen Planung geht es um den Aufbau und die Gestaltung des Textes. Eine wichtige Bedingung für die inhaltliche Planung ist das Thema des zu produzierenden Textes. Anders ausgedrückt würde man sagen, der Textproduzent muß eine erste Repräsentation des Themas aufbauen. Das Thema als Bedingung für inhaltliche Planung ist also in Relation zum Wissen des Textproduzenten zu betrachten. Ist das Thema für ihn eindeutig, dürfte weinger inhaltliche Planung notwendig sein, als 
wenn dem Textproduzenten nicht klar ist, was mit dem Thema zu verbinden ist. Was mit dem Thema zu verbinden ist, ist eine erste Aufgabe der inhaltlichen Planung.

Eine weitere wichtige Bedingung für die inhaltliche Planung sind die potentiellen Leser bzw. deren Erwartungen. Sie beeinflussen die Auswahl der Inhalte und auch die Form, in der diese Inhalte mitgeteilt werden. Der Textproduzent hat dabei das Vorwissen der Leser und deren sprachliche Fähigkeiten zu bedenken. Auch die Wortwahl ist hierdurch beeinflußt.

Auch die Funktion des Textes ist eine Bedingung für die inhaltliche Planung. Texte z.B., die überzeugen sollen, bedürfen bestimmter Argumentationsstrukturen, die nachvollziehbar sind.

Unter den Entscheidungen, die die inhaltliche Planung beeinflussen, kann das inhaltliche Wissen zum Thema wohl als grundlegende Voraussetzung betrachtet werden. So ist beispielsweise bei Abhandlungen zu empirischen Untersuchungen Konvention, zuerst den theoretischen Hintergrund auszuarbeiten, dann den Versuchsaufbau zu erläutern und folglich die Ergebnisse mitzuteilen.

Inhaltliche Planung kann rein mental ablaufen, oder der Textproduzent macht sich Notizen, um seine Gedächtniskapazität zu entlasten. Aber auch Planungsnotizen sind in einer Schaubild - oder Gliederungsform angefertigt.

In der Textproduktionsforschung wird des weiteren unterschieden zwischen Planung, die sich auf den ganzen Text bezieht (vgl. z.B. "global discourse plans" von Cooper \& Matsuhashi 1983 oder Makrostruktur bei van Dijk \& Kintsch 1983) und der Planung, die sich auf den einzelnen Satz bezieht ("sentence plans").

\subsubsection{Ausführung}

Ausführung umfaßt Orientierung, Generierung von Informationen, Formulierung (Vertextung) dieser Informationen gemäß dem Plan, Rezeption von geschriebenem Text und Evaluation des Textes.

\subsubsection{Orientierung}

Die Orientierung umfaßt zum einen alle vorbereiteten Handlungen zur Bereitstellung der notwendigen Materialien. Zum anderen zählt hierzu eine Situationsanalyse, d.h. die Repräsentation der situativen Bedingungen (z.B. Thema, Rezipient, Zeit usw.).

\subsubsection{Generierung}

Es werden einerseits Wissenselemente aus dem Langzeitgedächtnis abgerufen und andererseits externe Informationen für die Textproduktion verarbeitet. Die Generierung von Informationen wird von Hayes \& Flower (1980) als Assoziationsfolge gesehen, bei der unter Berücksichtigung der situativen Bedingungen Thema und Leser Informationen aus dem Langzeitgedächtnis abgerufen werden. 
Unterscheidet sich Textproduzieren bzw. Texte von Textproduzenten mit mehr bzw. weniger Wissen zu einem Thema? Ohne Zweifel hat die Vertrautheit mit dem Thema Einfluß auf die Verfügbarkeit von bedeutsamen Wissenselementen. Dies konnte in Untersuchungen, die den Einfluß von themenrelevantem Wissen auf Textproduzieren zum Gegenstand hatten, auch nachgewiesen werden (vgl. McCutchen 1986, Voss, Vesonder \& Spilich 1980, Witte \& Faigley 1981, Eigler et al. 1990). Die Wichtigkeit des Themas zeigt sich auch in dem in der Fachliteratur oft verwendeten Stichwort "Qualität": inwieweit sind einzelne Äußerungen, aus denen Texte bestehen, themabezogen? Als gute Texte werden in der Regel solche empfunden, die sich eng auf das gestaltete Thema beziehen, d.h. sich im Gesamtbereich für einen Leser durch Themenrelevanz auszeichnen. Molitor (1984: 27ff.) beispielsweise nennt einige Möglichkeiten, wie Generierung unterstüzt werden kann. Eine Möglichkeit ist die Benutzung eines Textschemas, das gemäß Anlaß und Funktion der Textproduktion abgerufen oder gebildet wird. Ein Textschema repräsentiert die Struktur des Textes und eröffnet sozusagen Leerstellen, die zu füllen sind. Steht dem Textproduzenten kein Textschema zur Verfügung, dann muß er die Struktur des Textes erst entwickeln. Weiterhin spielen metamnemonische Techniken eine Rolle, wie z.B. Gliedern von vorhandenem Material, Perspektivenwechsel, Analogien usw.

Beim Textproduzieren spielt auch die Verwendung von schriftlichen Informationen eine wichtige Rolle.

Generierung umfaßt somit zwei Bereiche: Abrufen von Wissen aus dem Langzeitgedächtnis und die Verarbeitung von externen Informationen. Das Wissen des Textproduzenten wird in Informationselemente im Arbeitsgedächtnis überführt und mit dem Thema verknüpft. Der Textproduzent entwickelt eine Repräsentation des Themas und damit eine erste Repräsentation des Textes.

\subsubsection{Formulierung}

Durch die Formulierung werden die generierten und durch die Planung organisierten Inhalte versprachlicht. Dabei handelt es sich um einen Übersetzungsvorgang von nichtsprachlichem semantischem Material in Text. Dieser Vorgang setzt sich aus einer Folge von Entscheidungen und Handlungen zusammen (vgl. Cooper \& Matsuhashi 1983). Einige Autoren betonen, daß schon bei der inhaltlichen Planung Sprache beteiligt sei. So greift z.B. Ludwig dazu das Konzept der inneren Sprache auf (Ludwig 1983: 60f.). Die Ergebnisse dieser innersprachlichen (Planungs-) Aktivitäten seien in semantischen Komplexen organisiert.

\subsubsection{Rezeption}

Durch Rezeption kann der Textproduzent die Repräsentation seines Textes erneuern. Auf der Grundlage der Textverarbeitungsforschung (z.B. Kintsch \& van Dijk 1978 ) ist - auf den zu produzierenden Text bezogen - anzunehmen, daß der Inhalt in Form einer Makrostruktur repräsentiert ist. 


\subsubsection{Evaluation}

Durch Evaluation wird ein Vergleich zwischen Intention und Realisation vorgenommen. Es gibt verschiedene Stufen der Evaluation (Rückkoppelung, Kontrolle, Bewertung), durch die geprüft wird, ob die Ziele verwirklicht sind (ob der Text den eigenen Intentionen entspricht, ob er kohärent ist usw.).

Zusammenfassend läßt sich sagen: Wenn wir schreiben oder sprechen, verschlüsseln wir konzeptuelle Inhalte in ein geordnetes Nacheinander von sprachlichen Einheiten. Vereinfacht gesagt, umfaßt dieser Vorgang folgende Prozeßebenen:

Auf der Stufe der Konzeptualisierung findet die Erstellung einer kognitiven Repräsentation des intendierten Äußerungsinhalts statt. Hier werden die Informationen ausgewählt, die ein Sprecher einem Hörer mit einer bestimmten Intention mitteilen will.

Die Stufe der Lexikalisierung involviert die Auswahl und Aktivierung der lexikalischen Einheiten, welche die konzeptuellen Inahlte ausdrücken sollen. Diese Ebene gehört bereits zum Prozeß der Formulierung. Ein weiterer Schritt ist die Erzeugung einer syntaktischen Struktur.

Auf der Stufe der phonologischen Enkodierung werden die lexikalischen Einheiten phonologisch spezifiziert.

Schließlich erfolgt die lautsprachliche Artikulation, d.h. die motorische Hervorbringung der sprachlichen Repräsentation.

Textproduzieren als kognitive und motorische Tätigkeit bedarf daher verschiedener Fähigkeiten, welche alle erlernt werden müssen.

\section{KOGNITIVE MODELLE DER TEXTPRODUKTION}

Kennzeichen der kognitiv orientierten Forschung zum Textproduzieren ist die Sicht des Textproduzierens als kognitiver Prozeß. Die Folge ist der Entwurf von kognitiven Prozeßmodellen (z.B. Hayes \& Flower 1980, Bereiter 1980, de Beaugrande 1984). Das sind auf einem generellen Niveau formulierte Vorstellungen hinsichtlich der am Textproduktionsprozeß beteiligten Komponenten (wie Lang- und Kurzzeitgedächtnis, Wissen u.ä.) und der Abfolge von Prozessen, die zwischen Wissen und Text vermitteln.

Sprachproduktionsmodelle werden in enger Interaktion zwischen Theorie und Empirie entwickelt. Theoretische Modelle erlauben die Ableitung von Hypothesen, die in experimentellen Untersuchungen überprüft werden können. Auf der Grundlage experimenteller Ergebnisse können die Modelle bestätigt oder modifiziert werden.

In der Textproduktionsforschung ist eine Trennung des Bereiches der gesprochenen Sprache von dem Bereich der geschriebenen Sprache meist die Regel. 
Gerade damit versäumt die Forschung jedoch, integrative Einsichten in den Sprachproduktionsprozeß zu gewinnen. Sprechen und Schreiben unterscheiden sich zwar in vielerlei Hinsicht. Sprechen - eher als ein Produkt der Evolution angesehen - ist in die Situation einer normalerweise mindestens dialogischen face-to-face Kommunikation eingebettet. Schreiben ist jedoch in eine eher monologische Situation eingebettet (vgl. Tannen 1985). Aus diesem Unterschied ergeben sich Konsequenzen für unterschiedliche Produktionsgeschwindigkeiten (vgl. Chafe 1977), für die Interpunktion oder für eine unterschiedliche Wortwahl (Hayes 1988). Natürlich ließe sich eine ganze Liste weiterer differenzierender Merkmale auflisten, die jedoch die Schlußfolgerung nicht rechtfertigen läßt, daß der Produktion gesprochener Sprache und der Produktion geschriebener Sprache völlig unterschiedliche kognitive Prozesse zugrunde liegen. Obwohl insbesondere Informationsquellen und Kontextbedingungen beim Schreiben und beim Sprechen teilweise von unterschiedlicher Relevanz sind, ist davon auszugehen, daß beim Sprechen und Schreiben eine große Schnittmenge zentraler kognitiver Produktionsprozesse vorhanden ist (van Dijk \& Kintsch 1983, Herrmann 1985, Bereiter \& Scardamalia 1987). Eine Verbindung beider Forschungsbereiche erscheint deshalb sinnvoll. Dies gilt gerade dann, wenn man auch die Genese der Schreibentwicklung berücksichtigt. Schreiben muß gelernt werden und die Fähigkeit, schriftliche Texte produzieren zu können, müssen Kinder auf der Grundlage ihrer mündlichen Sprachfähigkeit entwickeln. Eine naheliegende und die mündliche und schriftliche Textproduktionsforschung verbindende Fragestellung ist deshalb z.B.: "Welche kognitiven Fähigkeiten müssen zu den für die mündliche Sprachproduktion gelernten zusätzlich entwickelt werden, damit schriftliche Texte produziert werden können?" (vgl. Scardamalia \& Bereiter 1987).

Die Produktion geschriebener Sprache ist Gegenstand wissenschaftlicher Analysen erst etwa ab den siebziger Jahren unseres Jahrhunderts (z.B. Stallard 1974, Flower \& Hayes 1977 u.a.). ${ }^{2}$ Hingegen wurden die Probleme bei der mündlichen Produktion schon Ende des letzten Jahrhunderts untersucht, und zwar anhand mündlicher Fehlleistungen (Meringer \& Mayer 1895). Ab den 50er Jahren entstanden weitere Untersuchungen zur Produktion gesprochener Sprache (z.B. Goldman - Eisler 1951, 1954; Maclay \& Osgood 1959). Dies hatte zur Folge, daß die Theorieentwicklung im Bereich der mündlichen Textproduktion der Theorieentwicklung der schriftlichen Produktion weit voraus war. Dies ist erstaunlich, wenn man die tägliche Flut von geplanten und schließlich produzierten schriftlichen Publikationen berücksichtigt.

Wie schon erwähnt, ist die schriftliche Textproduktionsforschung eine verhältnismäßig junge Disziplin und die Theorieentwicklung in diesem Bereich beginnt erst in den achtziger Jahren mit den Arbeiten von Hayes und Flower (1980; Flower \&

2 In den achtziger Jahren sind die in der amerikanischen Forschung entwickelten Theorien und Modelle in deutschsprachigen Raum aufgenommen, kritisiert und weiterentwickelt worden. Vgl. z.B. Fischer \& Mandl 1980, Kluwe 1981, das Modell von Borkowski \& Krause 1985. 
Hayes 1981a). Das Ziel war zunächst festzustellen, ob verschiedene Phasen in der schriftlichen Textproduktion überhaupt zu erkennen sind. Fallstudien zu geübten Textproduzenten, die während der Textproduktion mündlich permanent darüber berichteten, worüber sie gerade nachdachten (lautes Denken), zeigten, daß Textproduktion ein rekursiver Prozeß ist.

Im folgenden werden wesentliche kognitive Modelle der schriftlichen Textproduktion dargestellt:

1. Das sogenannte rekursive Modell von Hayes \& Flower (1980), das zu einer Art Leitmodell geworden ist (fast in allen Arbeiten zum Textproduzieren wird darauf Bezug genommen). Es bildet den zentralen Rahmen der Schreibforschung.

2. Das am weitesten ausgearbeitete, stärker linguistisch orientierte Modell des parallelen Verarbeitens von de Beaugrande (1984).

3. Das Modell von Schreibstrategien von Bereiter (1980) bzw. von Bereiter \& Scardamalia $(1983,1985,1987)$, das sich auf Schreibproduktionsprozesse von Anfängern konzentriert.

4. Das Modell von Cooper \& Matsuhashi (1983) versucht zu erklären, welche Verarbeitungsschritte beim Übergang von der Planungsphase in die Übersetzungsphase im Produktionsprozeß notwendig sind.

\subsection{Das Modell von Hayes \& Flower}

Die Textproduktion wird in dem Modell von Hayes \& Flower (1980) als ein globaler zielgerichteter Prozeß im Sinne eines Problemlöseprozesses verstanden, wobei es zu einem Wechselspiel zwischen Intention und Realisation kommt. Die Zielgerichtetheit besteht in der Annäherung an die Zielvorstellungen hinsichtlich Thema, Leser und Form (im Sinne von Textschema). Dieser Zielgerichtetheit entspricht auf der Ebene des Handelns eine flexible Organisation der Prozesse wie Planen, Übertragen (Übersetzen) in schriftliche Form und Prüfen, die ihrerseits in weitere Subprozesse zerfallen. Diese Abfolge ist nicht einfach linear-sequentiell anzusehen, da es sich um ein rekursives Modell handelt: eine Phase der Planung wird von einer Phase der Übertragung (Übersetzens) und diese wiederum von einer Phase des Überarbeitens abgelöst. Ein kontinuierliches Durchlaufen dieser Phasen führt schließlich zu einem vom Textproduzenten gewünschten Textendprodukt. Die Voraussagen von Hayes \& Flower waren, daß zunächst Prozesse des Bereitstellens von Wissen, gelegentlich unterbrochen von Prozessen des Prüfens, vorherrschen, dann Prozesse des Organisierens und schließlich Prozesse des Übertragens, jeweils unterbrochen von Prozessen des Bereitstellens neuen Wissens und des Prüfens. Die Autoren arbeiten mit der Methode des lauten Denkens und der Protokollanalyse, um kognitive Prozesse beim Textproduzieren zu identifizieren. Aufgrund der ihrem Modell zugrundeliegenden 
empirischen Daten konzentrieren sie sich auf die Subprozesse der Planung und der Übersetzung.

Hayes \& Flower (1980) unterscheiden innerhalb der Textproduktion drei relevante Bereiche:

1. die Textproduktionssituation

2. das Langzeitgedächtnis des Textproduzenten und

3. die aktuelle Textproduktion (der Schreibprozeß selbst)

Zur Textproduktionssituation gehören mehrere Faktoren, von denen drei zentral sind. Der erste Faktor ist die Aufgabe, einen ganz bestimmten Text mit einem konkreten Thema zu verfassen. Darüber hinaus muß sich der Textproduzent an den spezifischen Lesern orientieren, für die er schreibt. Den dritten Faktor stellt der aktuell produzierte Text dar, d.h. der Textteil, der nach der bereits begonnenen Textproduktion zustande gekommen ist. Der produzierte Text ist nämlich die Grundlage für seine kohärete Fortführung.

Mit dem Langzeitgedächtnis ist das unterschiedliche Wissen (Sachwissen, Wissen über den sprachlichen und strukturellen Aufbau des Textes sowie über die Texttypen bzw. Textsorten, Wissen über Leser usw.) des Textproduzenten gemeint, in der Hauptsache wohl das Wissen über den Themenbereich, zu dem zu schreiben ist. Dieses Wissen ist der Ausgangspunkt für die weitere Verarbeitung. Zur Bewältigung einer bestimmten schriftlichen Aufgabe muß nun der Textproduzent all die dafür notwendigen Informationen aus seinem Langzeitgedächtnis abrufen. Somit ist Textproduktion ein zielgerichteter Problemlöseprozeß.

Der aktuellen schriftlichen Textproduktion, die nur eine Komponente des Modells darstellt, ordnen Hayes \& Flower drei Prozeßtypen zu:

- Planungsprozesse

- Übersetzungsprozesse

- Überarbeitungsprozesse

Planungsprozesse eröffnen die Chance, nicht an der Vielfalt heterogener Anforderungen zu scheitern. Pläne reduzieren - zunächst nur umrißhaft - auf das Wesentliche. Planungsprozesse dienen dazu, eine interne mentale Repräsentation des Wissens aufzubauen, das in einen Text transformiert werden soll. Welche Struktur und welche Komponenten dieser internen Wissensrepräsentation zugrunde liegen, bleibt jedoch bei Hayes \& Flower uneindeutig (vgl. Flower \& Hayes 1981a: 372). Einerseits behaupten sie nämlich, daß das Wissen in Form von Propositionen gespeichert ist, andererseits aber sagen sie, daß Konzepte, Relationen und Attribute, die eigentlich die Wissensstruktur bilden, in Komplexen von Netzwerken oder Bildern organisiert sind (Hayes \& Flower 1980: 15).

Hayes \& Flower unterscheiden drei Planungsprozesse: Generierungsproze $\beta$, Organisationsproze $\beta$ und Beurteilungsprozeß. 
Die Aufgabe des Generierungsprozesses besteht in dem Abrufen von notwendigen Informationen aus dem Langzeitgedächtnis. Der Textproduzent generiert ein Netzwerk von Ideen, das weit über den tatsächlich produzierten Textinhalt hinausgehen kann. Ergebnis dieses Prozesses sind "single words or sentence fragments, although they may sometimes be complete sentences." (Hayes \& Flower 1980: 15ff.).

Der Organisationsproze $\beta$ linearisiert die im Hinblick auf das Thema abgerufenen Informationen entsprechend ihrer Wichtigkeit in einem Schreibplan.

Der Beurteilungsproze $\beta$ übernimmt eine Selektionsfunktion. Er wählt zwischen den generierten und organisierten Informationen solche Informationen aus, die für die Schreibaufgabe besonders geeignet sind und gleichzeitig den Sprachkonventionen hinsichtlich schriftlicher Texte genügen. So wird während der Planungsphase eine mentale Repräsentation des zu produzierenden Textes aufgebaut.

Mit Hilfe des Übersetzungsprozesses wird die mentale Repräsentation in Sprache transformiert. Die Aufgabe des Übersetzungsprozesses ist es nun, der linearisierten propositionalen Repräsentation sprachliche Korrelate zuzuordnen. Eben an dieser Stelle wird der oben angedeutete Widerspruch in den Arbeiten von Hayes \& Flower offensichtlich. Wenn schon innerhalb der Planungsphase der Generierungsprozeß einen sprachlichen Output erzeugt, nämlich einzelne Wörter, Satzfragmente oder auch ganze Sätze, dann wäre der Übersetzungsprozeß nicht mehr notwenig. Es ist deshalb davon auszugehen, daß das Ergebnis des Generierungsprozesses nicht einzelne Wörter, Satzfragmente oder Sätze sind, sondern atomare und komplexe Propositionen, die während des Übersetzungsprozesses in Sprache übersetzt werden.

Unter Überarbeitungsprozessen verstehen Hayes \& Flower Produktionsschritte, die die Qualität des schon produzierten Textes sowohl in inhaltlicher als auch in stilistischer Hinsicht verbessern oder auch verändern. In diese Prozesse sind auch Leseprozesse des Textproduzenten eingebettet, und zwar mit dem Ziel, einen Vergleich zwischen dem geschriebenen und dem intendierten (geplanten) Text zu ermöglichen. Treten Diskrepanzen auf, dann können mittels Korrekturen rekursive Anpassungen vorgenommen werden.

Die Prozesse der Planung, Übersetzung und Überarbeitung sind in diesem Modell hierarchisch organisiert und können nach Hayes \& Flower parallel arbeiten. Dabei gehen die Autoren davon aus, daß die sprachliche Realisierung eines Textes direkt von der vom Textproduzenten aufgebauten mentalen Repräsentation abhängt, d.h. die Repräsentation wird von Propositionen schrittweise in schriftlich-sprachliche Form überführt. Qualitative Angaben darüber, wie der Übersetzungsprozeß von einem mental geplanten zu einem sprachlich realisierten Text vonstatten geht, fehlen bei Hayes \& Flower gänzlich. Mit anderen Worten, die der Introspektion nicht zugänglichen Prozesse wie z.B. die Übergabe semantischer Informationen an den von Hayes \& Flower angenommenen Übersetzungsprozeß stehen nicht im Mitelpunkt ihrer Forschungsarbeiten. 
Das Modell selbst wird von den Autoren als provisorisch eingeschätzt, als Leitfaden für Forschung und als Ausgangspunkt bei der Suche nach stärker ausgearbeiteten Modellen. Wissenschaftlich gesehen hat es den Charakter eines Rahmenmodells.

Die Autoren sagen: "Our model is a model of competent writers" (1980: 29). Darin zeigt sich eine weitere Beschränkung des Modells: Auf seinem Hintergrund mögen Mängel des Schreibens von Anfängern faßbar werden - es gibt aber keinerlei Anhaltspunkte, wie aus Schreibanfängern Schreibexperten werden.

\subsection{Das Modell von de Beaugrande}

Der Autor, von der Linguistik herkommend, versucht in das Problem des Textproduzierens den kognitiven Ansatz und dessen Ergebnisse zu integrieren. Bei der Einschätzung der Forschungslage ist de Beaugrande der Meinung, daß Textproduzieren in Anbetracht seiner Komplexität sinnvoll nur erforscht werden kann, wenn vielfältige Veriablen in Modelle integriert werden und deren Interaktion zum Gegenstand der Forschung gemacht wird. So hebt sein parallelstage interaction model die Parallelverarbeitung und die Interaktion der Produktionsphasen hervor. Außerdem weist es die Kapazitätsbeschränkungen des Kurzzeitgedächtnisses eines Autors als physiologisches Argument aus, weshalb die Informationsmenge, die sprachlich realisiert werden kann, die Satzebene in der Regel nicht überschreitet. Sein Ziel ist eine Wissenschaft des Textschreibens (science of composing, theory of writing); diese sieht er eingebettet in eine allgemeinere Theorie des Textproduzierens (die mündlichem und schriftlichem Textproduzieren übergeordnet ist) und diese wiederum in Theorien des kognitiven Verarbeitens (cognitive processing) einerseits und der Kommunikation und Interaktion andererseits. Der Ebene des Textproduzierens ist das Sprachsystem zugeordnet, das seinerseits wieder Subsysteme umfaßt (bezogen auf Laute, Wörter, syntaktische Muster, Begriffe, Vorstellungen usw.), die gleichzeitig operieren und deren Interaktion das Handeln des Gesamtsystems konstituiert. Im Licht der übergeordneten Theorien (des kognitiven Verarbeitens wie der Kommunikation) erhalten linguistische Syntax, Semantik und Pragmatik eine spezifische Deutung: linguistische Syntax als Spezialfall linearen Verarbeitens, Semantik als Spezialfall begrifflichen Verarbeitens und Pragmatik als Spezialfall von Zielplanung. Zwei Tendenzen sind zu beobachten: Steigerung von Komplexität und ihre Reduktion durch Systematisierung.

Das Modell umfaßt mehrere Ebenen, auf denen sukzessiv, aber auch parallel operiert werden kann, wenn der Schwerpunkt jeweils auf einer Ebene liegen dürfte (s. Abb.1). Von "höheren" (d.h. logisch betrachtet: nachgeordneten) Ebenen kann auf "tiefere" Ebenen zurückgekehrt werden, wenn es die Entwicklung erforderlich macht im Modell symbolisch durch die Zick-Zack-Verbindungen der Ebenen. Auf der tiefsten Ebene - goal-planning - vollzieht sich die Festlegung des Ziels, das mit diesem Text 
erreicht werden soll, und des Plans, wie dieses Ziel erreicht werden soll. Auf der nächsten Ebene - ideation - kommt es zur Fixierung der "idea". Darunter versteht de Beaugrande:

"a configuration of conceptual content that acts as a control center for building the text-world model (the total configuration of knowledge activated for processing the text)."

(de Beaugrande 1984: 109)

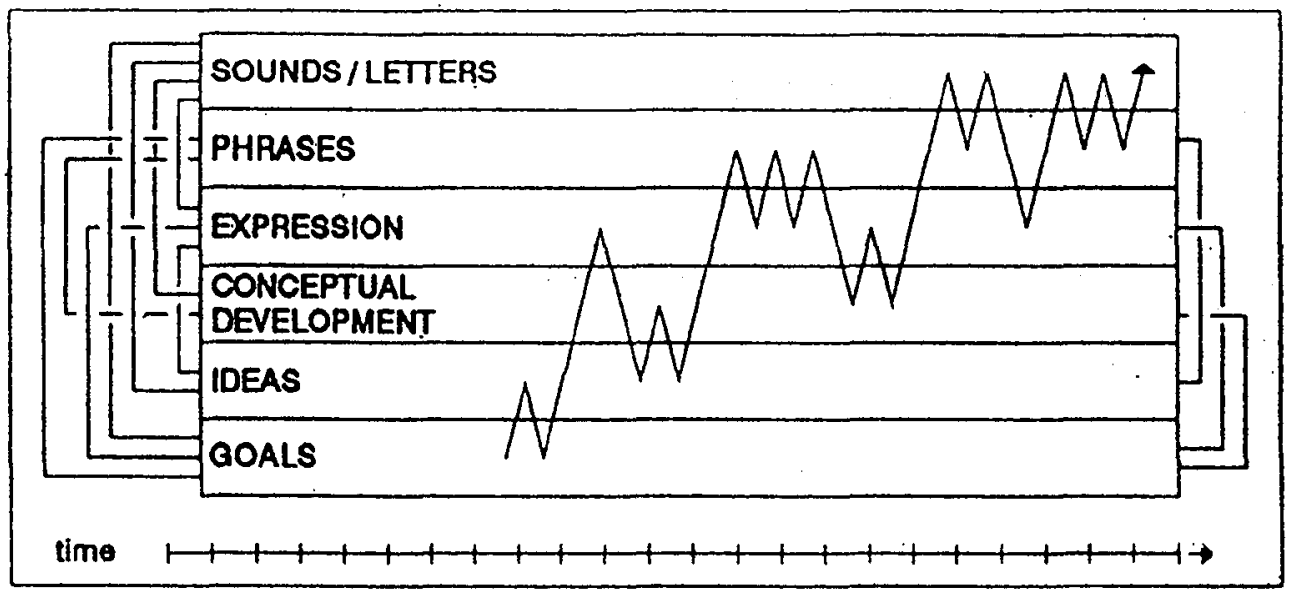

Abb. 1 Das Modell interaktiven, parallelen Verarbeitens (zitiert nach de Beaugrande 1984: 106)

Das besagt: Im Hinblick auf das Thema wird ein noch recht allgemeiner begrifflicher Rahmen entworfen, der dann eine gewisse Kontrollfunktion übernimmt, nämlich welches Wissen im Fortgang zu aktivieren ist, um den Rahmen auszufüllen, bzw. welches - sich möglicherweise assoziativ einstellende - Wissen auszuscheiden ist. Auf der nächsten Ebene - conceptual development - geschieht nun diese Anreicherung, bis ein detailliertes "text-world model" entsteht (de Beaugrande 1984: 129). Es folgen die Ebenen expression, auf der die gedanklichen Konfigurationen sprachlich gefaßt werden, dann phrase linearization und sound/letter linearization, auf denen die endgültige Überführung in die Form eines zusammenhängenden Textes vollzogen wird. Es handelt sich nun um kein lineares bzw. serielles Modell; vielmehr stellen die Ebenen nur funktionale Einheiten und keineswegs ausschließlich zeitliche Einheiten dar. Von der Ebene der Entwicklung der Gedanken kann auf die der Entwicklung des Rahmens zurückgegangen werden, wenn sich dieser bei der Weiterarbeit als inkonsistent erweisen sollte, oder: Von der Ebene des textlichen Linearisierens kann auf die der Entwicklung der Gedanken zurückgegangen werden, wenn sich beim Versuch des Linearisierens zeigt, daß die bisherige gedankliche Arbeit unzureichend war, sich z.B. 
Lücken oder gar Widersprüche zeigen. Das heißt: De Beaugrande nimmt wie Hayes \& Flower (1980) die Möglichkeit einer sehr flexiblen Organisation der einzelnen Teilprozesse an.

De Beaugrande versucht zu erhellen, was mit dem Wissen beim Textproduzieren geschieht. Dabei weist er auf die Funktion des Gedächtnisses als Wissensspeicher beim Textproduzieren hin. Bei jedem Textproduzieren wird Wissen aktiviert und zugleich aktualisiert. Aktualisierung meint, daß - bezogen auf die Textproduktionssituation (Thema, Zweck) - entsprechende Auswahlen getroffen werden und dieses ausgewählte Wissen thema- und zweckangemessen in Zusammenhang gebracht wird. Ganz allgemein wird daher erwartet, daß Wissen zu einem Thema einen Einfluß auf den Prozeß und damit auf das Produkt von Schreiben hat.

- Das Gedächtnis fungiert als Wissensspeicher.

- Nimmt der Schreiber einen kleinen Ausschnitt des Textes wahr (nämlich den gerade geschriebenen), so hat er rückwärts- und vorwärtsgewandt eine Repräsentation des geschriebenen Textes. Das hat erhebliche Konsequenzen:

"Hence, the text producer makes only fleeting contact with the surface text, and carries out most activities upon a mental representation. The latter's format differs from that of the surface text in important ways: being not just linear, but also hierarchical (...); including materials not expressed in the text (...).

(de Beaugrande 1984: 128)

In der Begrifflichkeit des Modells bedeutet das folgendes: In der "ideation"-Phase kommt es zum Entwurf eines gedanklichen Rahmens für den Text, der die weitere gedankliche Differenzierung wie auch die Prozesse der "expression"- und "linearization" - Phase steuert, der dabei aber in einer Art Makrostrukturbildung (vgl. van Dijk \& Kintsch 1983) mit den auf der Textebene erreichten Differenzierungen angereichert wird. Das besagt: So wie der Schreiber den künftigen Text nicht im Detail, sondern in Form einer "configuration of conceptual content" (de Beaugrande 1984: 109) antizipiert, so verfügt er auch nach dem Schreiben nicht über eine gedächtnismäßige Repräsentation des geschriebenen Textes im Detail, sondern über eine Makrostruktur, die während des Schreibens erzeugt wird.

Während des Produzierens eines Textes wird ständig und zwar in sehr unterschiedlicher Weise mit Wissen umgegangen. Wissen wird aus dem Gedächtnis aktiviert, wird - situationsbezogen - aktualisiert.

Hier lassen sich Verbindungen zum Textverarbeiten und Lernen mit Texten, aber auch zum Wahrnehmen herstellen: Jeweils wird auf Wissen zurückgegriffen, auf "our prior model of the world" (de Beuagrande 1984: 3), jeweils handelt es sich um Nutzung eigenen Wissens, das sich entwickelt, indem dieses Wissen und die spezifischen 
Gegebenheiten der Situation, sei es in Prozessen des Textverarbeitens, sei es in Prozessen des Wahrnehmens, vermittelt werden.

Auch in diesem Modell fehlen prozedurale Angaben darüber, wie ein Ausschnitt einer nichtsprachlichen konzeptuellen Repräsentation als Satz, von dem der Produzent zu Beginn der Produktion normalerweise noch nicht weiß, wie er syntaktisch aufgebaut oder wann er zu Ende ist, inkrementell produziert wird.

\subsection{Das Modell von Bereiter und von Bereiter \& Scardamalia}

Auch Bereiter (1980) geht bei seinen Überlegungen von kognitiven Theorien aus. Im Zentrum seiner Forschung steht die Frage, welche kognitiven Strategien bei der schriftlichen Textproduktion angewendet werden und wie diese angepaßt sind an die jeweils begrenzte Informationsverarbeitungskapazität. Er (1980) entwirft ein Prozeßmodell, das folgende Komponenten umfaßt: auf einer obersten Ebene ein "exekutives Schema", das den Schreibprozeß als ganzen unter Berücksichtigung von Zwecken (d. h. in der Sprache von Hayes \& Flower (1980): von Thema und potentiellen Lesern) steuert; auf der darunterliegenden Ebene steuern "Textschemata", den Schreibprozeß hinsichtlich der Textform (diese hängt wiederum von Textsorte $a b$ ), dann einen "Inhaltsverarbeiter" ("content processor", Bereiter 1980: 79, vergleichbar den Prozessen "Bereitstellen von Wissen" und "Organisieren") und schließlich einen "Sprachverarbeiter" ("language processor", ebenda S. 79), vergleichbar dem ProzeB "Übertragen" bei Hayes \& Flower (1980). Auch zu dem Modell von de Beaugrande (1984) lassen sich entsprechende Beziehungen herstellen. Auf dem Hintergrund dieses hierarchischen Modells wird der Schreibprozeß selbst als ein Wechselspiel von absteigenden und von aufsteigenden Prozessen angesehen (aufsteigende Prozesse bedeuten die Aktivierung der höher liegenden Hierarchieebenen). Die sich entwickelnde Fähigkeit zur Bewältigung der Verarbeitungsüberlastung beim Schreiben erklärt Bereiter (1980) mit Annahmen van Pascual-Leone \& Smith 1969, nach denen mit dem Alter zunehmend - eine größere Zahl von Tätigkeiten koordiniert wird. So praktizieren z.B. Schreibanfänger im Vergleich mit Schreibexperten ein strukturell einfacheres Schreiben. Bei ihnen stehen Probleme auf niederen Ebenen im Vordergrund, weil zunächst überhaupt etwas zu Papier gebracht werden muß. Die Anfänger praktizieren eine Schreibstrategie, die Gesichtspunkte höherer Ebenen - wie z.B. potentielle Leser oder Textschemata - nicht einzubeziehen vermag. Bereiter unterscheidet "associative writing": der Schreiber schreibt, solange ihm etwas einfällt. Wird dann das Wissen von den Schreibkonventionen, von der Orthographie bis hin zur Syntax integriert, konstituiert sich die Schreibstrategie "performative writing". Der nächste Entwicklungsschritt führt vom performativen zum kommunikativen Schreiben ("communicative writing"). Erst jetzt wird der potentielle Leser berücksichtigt. Das 
kommunikative Schreiben ist die wohl verbreitetste Form des alltäglichen Schreibens, in dem nun Inhalt, Form und Leser in gleicher Weise den Schreibprozeß steuern.

Wenn sich die Schreibfähigkeiten mit den beim Leser realisierten bewertenden Fähigkeiten verbinden, entsteht ein sogenannter "feedback loop" von Schreiben und Lesen, in dem das Produkt des Schreibens, also der Text, unter Standards des Schreibers gesehen, seiner kritischen Bewertung in formaler als auch inhaltlich-logischer Hinsicht unterworfen wird. Der Text wird nun als etwas empfunden, das zu gestalten ist, d.h. Textproduktion wird nicht mehr nur als instrumentelle Fähigkeit, etwas mitzuteilen, gesehen, sondern weit stärker als eine produktive Tätigkeit. Dabei bleibt auch diese Schreibstrategie - "unified writing" genannt - noch vorwiegend textbezogen.

Das ändert sich, wenn noch ein letzter Schritt in der Schreibentwicklung vollzogen wird, wenn erkannt wird, daß der für "unified writing" konstitutive "writing-reading feedback loop" nicht nur zu einem verbesserten Text, sondern auch zu einem verbesserten Verstehen ("improved understanding", ebenda S. 89) führt. Denken und Wissen können durch die kognitiven Konsequenzen des Schreibens (der Textproduktion) angereichert werden. Insofern kommt in diesem Schreiben die epistemische, d.h. Wissen-entwickelnde Funktion des Schreibens voll zum Tragen. Wann immer Texte produziert werden, ob in der Form des "performative writing", des "communicative writing" oder des "unified writing", wird nicht nur auf Wissen zurückgegriffen, sondern wird dieses Wissen allein schon durch den Einbezug in einen Textproduktionsprozeß modifiziert. Was de Beaugrande (1984) als Aktivierung von Wissen, die immer eine Aktivierung im Blick auf den Schreibzweck ist, zu fassen versuchte, erscheint bei Bereiter (1980) als epistemische Funktion des Schreibens.

Bereiter hat ein hierarchisch-rekursives Modell entwickelt, das, verglichen mit dem Modell von Hayes \& Flower (1980), viel stärker theoretisch fundiert ist.

Bereiter steht in der Tradition vieler Dichter und Schriftsteller, aber auch Wissenschaftler wie z.B. Wygotski (1934), Luria (1973) und Bruner \& Olson (1976): daß sich Wissen durch Schreiben, d.h. durch diese spezifische Form der Versprachlichung, entwickeln und damit auch verändern kann.

\section{Das Modell von Bereiter \& Scardamalia (1983, 1985, 1987)}

In ihrem Modell der schriflichen Textproduktion gehen die Autoren einen methodisch und theoretisch anderen Weg als z.B. Hayes \& Flower (1980), Flower \& Hayes (1980, 1981, 1984) und Cooper \& Matsuhashi (1983). Sie versuchen, beim Schreiben verwendete Fähigkeiten und Strategien zu erkennen. Dabei vergleichen sie Schreibproduktionsprozesse geübter Textproduzenten mit Produktionsprozessen von Anfängern. Ihr Interesse konzentriert sich jedoch auf den Schreibanfänger, der Schreibfähigkeiten eines geübten Textproduzenten erst noch erlernen muß. Sie 
untersuchen also, wie Kinder beim Schreiben von Texten vorgehen. Dieser Forschungsbereich findet zunehmend Beachtung (s. z.B. McCutchen 1986).

Der gemeinsame Nenner der Produktionsmodelle für geschriebene Sprache besteht in der Tatsache, daß die Produzenten ihr Wissen im Produktionsprozeß transformieren können. Bereiter \& Scardamalia nennen diese Modelle deshalb auch "knowledge transforming-Modelle". Der geübte Textproduzent kann mit Faktoren wie Ziele, Texttyp, potentielle Leser bewußt umgehen. Die Kinder dagegen verfügen nicht über die Fähigkeit zu dieser Art Reflexion. Darunter verstehen die Autoren z.B. konkrete Wirkungen, die der Text bei bestimmten Lesern hervorrufen soll. Potentielle Leser werden von Kindern nicht antizipiert. Kinder entwickeln während der Textproduktion keine über die Schreibaufgabe hinausgehenden individuellen Ziele. Deshalb setzen Bereiter \& Scardamalia $(1985,1987)$ den "knowledge transform- ing"-Modellen ein "knowledge telling"-Modell gegenüber, das den Produktionsprozeß bei Kindern beschreiben soll.

Das Modell geht davon aus, daß Kinder im Produktionsprozeß lediglich drei Informationsquellen benutzen können, um einen Text zu produzieren. Diese sind:

- das Thema des Textes

- Diskursschemata und

- der bereits produzierte Text

Diese Schlußfolgerung ziehen die Autoren aus eigenen empirischen Untersuchungen.

Das Thema dient als Stimulus, um Informationen schnell aus dem Wissen abzurufen. Diese Informationen können jedoch nicht wie bei geübten Textproduzenten beliebig angeordnet werden. Sie werden nämlich so aktiviert, wie sie in der Wissensrepräsentation der Kinder mental repräsentiert sind.

Unter Diskursschemata verstehen Bereiter \& Scardamalia Wissen über den Aufbau verschiedener Textsorten. Kinder verfügen nicht nur über Wissen über den Aufbau narrativer Texte, sondern auch Wissen über den Aufbau von Wegbeschreibungen oder Erörterungen.

Die Funktion des bereits geschriebenen Textes ist es, weitere Informationen aus der Wissensrepräsentation der Kinder abzurufen.

Globale Planungsprozesse, die den Aufbau des gesamten Textes partiell vorwegnehmen, treten bei Kindern nicht auf: "... the topic would give rise to a first utterance, something in the first utterance would provide a cue for a second, and so on." (Bereiter \& Scardamalia 1987: 343). Das Fehlen globaler Prozesse erklärt, warum von Kindern produzierte Texte auf der Ebene zweier Sätze Kohärenz aufweisen (lokale Kohärenz), häufig aber nicht auf der gesamten Textebene (globale Kohärenz) (vgl. McCutchen \& Perfetti 1982, Karmiloff-Smith 1985). Das bedeutet, daß die Kinder nicht ausschließlich textrelevante Informationen aktivieren. Bereiter \&Scardamalia nehmen deshalb eine Prüfphase an, die es den Kindern ermöglicht, solche Informationen vor der 
Produktion auszusondern, die nicht mit der Textstruktur und dem Thema des Textes kompatibel sind. Diese Prüfphase ist die einzige Komponente im Modell, die eine bewußte Informationsselektion im Produktionsprozeß zuläßt.

Textproduktion unter der Perspektive einer Wissenswiedergabe-Strategie eines Schreibanfängers unterscheidet sich zentral von einer Wissenstransformations-Strategie eines geübten Schreibers. Erstens produzieren Kinder den Text nicht als Instrument, um individuelle Ziele zu realisieren oder um bestimmte Wirkungen und Einstellungen bei Lesern zu erzeugen. Zweitens ist Wissen wiedergeben eine routinisierte und bewußt nur bedingt vom Textproduzenten steuerbare Strategie. Wissen transformieren ist hingegen ein Problemlöseprozeß, währenddessen der Produzent rational und rekursiv alle Informationsquellen auswertet und erst dann einen Text produziert, der nach individuellen Einschätzung den Anforderungen der Schreibsituation genügt.

Bereiter \& Scardamalia heben deshalb hervor, daß schriftliche Textproduktion im wesentlichen ein kognitiver Prozeß ist, bei dem das Operieren mit mentalen Wissensrepräsentationen über Sprache, Ideen, Ziele und Textstrukturen im Vordergrund steht.

Das knowledge telling-Modell bleibt auf einer relativ globalen Beschreibungsebene. Wie aufgrund von thematischem und textstrukturellem Wissen selektierte Informationen in einen konkreten Text transformiert werden, bleibt völlig ungeklärt (vgl. Bereiter \& Scardamalia 1987: 24ff.).

Bereiter \& Scardamalias Ziel ist es, aus Differenzen, die es zwischen den Fähigkeiten gibt, Wissen lediglich wiedergeben oder Wissen transformieren zu können, Möglichkeiten abzuleiten, um Kinder in pädagogischen Lehr- und Lernprozessen an Fähigkeiten geübter Schreiber heranzuführen.

$\mathrm{Daß}$ eine Steigerung reflexiver Prozesse bei Kindern zumindest auf einer lokalen Ebene zu inhaltlich besseren Texten führen kann, zeigen Scardamalia, Bereiter und Steinbach (1984). In dieser Untersuchung trainierten Kinder während der Textproduktion einen inneren Dialog zu führen, also zu jedem Argument ein Gegenargument zu finden und dann abzuwägen, ob das Gemeinte dem Geschriebenen entspricht. Nach einer Trainingsphase waren die Texte der Experimentalgruppe inhaltlich wesentlich differenzierter als die der Kontrollgruppe (vgl. Bereiter \& Scardamalia 1987).

Schreibanfänger haben gegenüber geübten Schreibern feststellbare Defizite, über die inhaltliche Planung des Textes zu reflektieren. Bereiter \& Scardamalia zeigen, daß diese Fähigkeit lernbar ist. Wird diese Fähigkeit angewendet, dann verbessert sich die inhaltliche Qualität der Texte von Anfängern deutlich. Die zentrale Ausrichtung des Modells liegt somit aber ebenfalls auf bewußt steuer- und wahrnehmbaren Schreibprozessen und nicht auf der Schnittstelle zwischen der Textplanung und seiner sprachlichen Realisierung. 


\subsection{Das Modell von Cooper \& Matsuhashi}

Im Gegensatz zu Hayes \& Flower und Bereiter \& Scardamalia versuchen Cooper \& Matsuhashi, Übersetzungsprozesse auf der Satzebene zu spezifizieren. Dabei unterscheiden sie das Erstellen von globalen Diskursplänen, den Aufbau von Satzplänen und Schreibausführungshandlungen. Ihr Modell stellt somit ein top-down ausgerichtetes Stufenmodell dar.

Im Rahmen des Aufbaus von globalen Diskursplänen muß der Textproduzent zunächst bestimmen, welche Ziele und Zwecke er mit dem zu schreibenden Text erreichen möchte. Darüber hinaus müssen die Leser und der Texttyp berücksichtigt werden. Der Texttyp impliziert immer die Entscheidung für eine spezifische Textstruktur. Globale Diskurspläne stecken Rahmenbedingungen ab, an denen sich Satzproduktionsprozesse orientieren. Welche Verarbeitungsschritte auf der Satzebene bei der Produktion notwendig sind, beschreiben Cooper \& Matsuhashi als Satzplanung, und zwar im Rahmen von acht Schritten.

In einem ersten Schritt wird eine Proposition formuliert. Dafür müssen bestimmte Informationen aus dem Langzeitgedächtnis abgerufen werden. Sie werden dann in einer Prädikat-Argument-Struktur geordnet und einem die Diskursintention realisierenden Sprechakt zugeordnet. Im Anschluß daran wird das Thema einer Proposition bestimmt. Ferner wird die Proposition mit dem vorausgehenden sprachlichen Kontext verbunden und folglich werden der Prädikat-Argument-Struktur lexikalische Einträge zugewiesen (in Form von Wortstammformen). Erst in einem siebten Schritt wird die Proposition in einen grammatikalischen Satz transformiert und die lexikalischen Einträge flektiert. In dem letzten Verarbeitungsschritt wird das Verarbeitungsresultat, d.h. der Satz im Kurzzeitgedächtnis gespeichert, bis er geschrieben ist. Die Satzplanungsprozesse sind hier abgeschlossen. Der Produzent beginnt mit Schreibausführungshandlungen.

Cooper \& Matsuhashi gehen davon aus, daß Schreibausfïhrungshandlungen eines geübten Textproduzenten so trainiert sind, daß sie stark routinisiert sind und im Schreibprozeß die kognitive Verarbeitung des Produzenten nur wenig belasten.

Das Modell von Cooper \& Matsuhashi konzipiert den Schreibprozeß unter Berücksichtigung globaler Diskurspläne als sequentielle Planung und Realisierung einzelner Propositionen: der Prädikat-Argument-Struktur werden aus dem Lexikon Wörter zugeordnet und die mentale Repräsentation wird Proposition für Proposition als Text geschrieben. Die syntaktische Realisierung folgt dabei der semantischen Repräsentation. Wie die Transformation einer propositionalen Struktur in einen schreibund schließlich lesbaren Satz erfolgt, bleibt in dem Modell wiederum unterbestimmt. ${ }^{3}$ 
Diese sequentielle und wissentlich idealisierte Abfolge einzelner Planungsschritte auf der Satzebene betrachten die Autoren selbst als idealisiert. Problematisch ist in ihrem Modell die Annahme, daß Texte bzw. Sätze propositionsweise geplant und auch propositionsweise geschrieben werden. Der zeitliche on-line Verlauf der Struktur von Propositionen ist nicht identisch mit der Struktur geschriebener Sätze. Eine Transformation der semantischen propositionalen Repräsentation in eine Satzstruktur ist somit notwendig. Trotzdem kann dieses Modell zu einer diferenzierteren Theorie des Schreibprozesses beitragen.

\section{EMPIRISCHE METHODEN DER SPRACHPRODUKTIONS- FORSCHUNG}

In der kognitiv orientierten Textproduktionsforschung versucht man, die Determinantien des Produktionsprozesses mit Hilfe experimenteller Studien aufzudecken. Die Frage ist, ob es möglich ist - aufgrund der empirischen Untersuchungen und über interpretative Zuordnungen hinausgehend - die Annahmen hinsichtlich der Prozesse zu überprüfen.

Die Vielschichtigkeit der Schreibproduktionsprozesse macht es notwendig, das Textproduzieren aus den unterschiedlichen Perspektiven und mit verschiedenen Methoden zu untersuchen. Diese sind:

- Pausen

- normale vs. pathologische (z.B. aphasische) Sprachproduktionsfehler

- die Methode des lauten Denkens (verbale Protokollanalyse)

- normale Schreibfehler vs. pathologische (z.B. agraphische) Schreibstörungen

- Revisionen

Von zentraler Bedeutung sowohl in der mündlichen als auch in der schriftlichen Produktionsforschung ist die Methode der Auswertung von Pausenpositionen und Pausenlängen während der Textproduktion. Viele Untersuchungen bedienen sich dieser Methode der on-line Erhebung (vgl. Goldman-Eisler 1951, Maclay \& Osgood 1959, Butterworth 1975). Bei der Messung von Pausen wird die Position des Wortes innerhalb eines Satzes bzw. Textes in Beziehung gesetzt zur Länge der Pause, die der Produktion dieses Wortes vorausgeht. Systamtische Beziehungen zwischen der Pausenlänge einerseits und der Wortposition andererseits ermöglichen dabei unmittelbare Einblicke in aktuell ablaufende Prozesse der Planung und Produktion von Texten.

Der Produktion von Fehlern liegt eine Regelhaftigkeit der Interferenzbildung zwischen Planungseinheiten unterschiedlichster Komplexität (Phrasen, Wörter, Morpheme, Silben, Grapheme/Phoneme) zugrunde. Die (Methode der) Untersuchung von Sprache (s. z.B. Fromkin 1973, 1988) versucht aufgrund dieser Regelhaftigkeit zu bestimmen, wann welche Planungseinheiten im Produktionsprozeß relevant werden. 
Bei der Methode des lauten Denkens (s. z.B. Hayes \& Flower 1980, Flower \& Hayes 1981) sollen die Textproduzenten über ihre Gedanken vor und während der Textproduktion mündlich berichten.

In den Untersuchungen von Schreibstörungen geht es nicht um die Analyse der freien Textproduktion, sondern um die bei diesen Menschen noch vorhandenen Fähigkeiten, Schreibprogramme für einzelne Wörter generieren und diese aktivierten Programme motorisch ausführen zu können.

In den Untersuchungen von Revisionen stehen Überarbeitungsprozesse im Vordergrund.

Im folgenden soll nur die Pausen-Methode eingehender dargestellt werden.

\subsection{Schreibpausen}

Insbesondere die Analyse von Pausen im Sprachproduktionsprozeß stand eine Zeitlang im Vordergrund der Forschung. Mit dem Einbezug dieser Methode in die linguistische und kognitive Sprachforschung wurde nämlich eine Änderung der Betrachtungsweise vollzogen: von der in den bisherigen Überlegungen vorherrschenden produktorientierten Betrachtung zu einer mehr prozeßorientierten. Die Pausen-Methode besteht - wie schon erwähnt - in der Messung von Pausen, die mündlichen und schriftlichen Äußerungen vorausgehen. Dabei stellen sich einige zentrale Fragen wie z.B.:

- An welchen Stellen treten Pausen auf?

- Wie lang sind Pausen an verschiedenen Textstellen?

- Kann aus den Zusammenhängen zwischen Pausenpositionen und Pausenlängen auf eine Art kognitiven Rhytmus zwischen Planungs- und Schreib/Artikulationsprozessen geschlossen werden?

Viele Untersuchungen weisen darauf hin, daß das Vorkommen von Pausen und Verzögerungselementen (wie $h m$ und $a h h$ ) oft an Satzgrenzen zu beobachten ist. Darum wurde als grundlegende Planungseinheit der Satz angesehen. Eine Korrelation zwischen Pausen und syntaktischer Komplexität läßt sich aber nicht feststellen. Vielmehr scheint die Pausenmenge eher von der konzeptuellen Komplexität der Äußerung abzuhängen (s. Butterworth 1980).

Im Bereich der schriftlichen Textproduktionsforschung gibt es im Vergleich mit der mündlichen Sprachproduktionsforschung wenigere Studien. Als eine erste empirische Untersuchung zur Überprüfung der konstatierten Beziehung zwischen Pausenpositionen, Pausenlängen und Planung beim Schreiben kann die Arbeit von Matsuhashi (1981) angesehen werden. In ihrem Artikel Explorations in the Real-time production of Written-Discourse (1982) beabsichtigt sie nicht nur ein integratives Modell des Textproduktionsprozesses, sondern auch Voraussetzungen für die Überprüfung entsprechender Hypothesen bereitzustellen. Integrativ soll das Modell insofern sein, als es - vergleichbar den anderen kognitiven Modellen - die starre 
Abfolge von Subprozessen im linearen Modell zugunsten flexibler Verknüpfungen funktional durchaus unterscheidbarer Teilprozesse auflöst. Der leitende Gesichtspunkt ihrer Arbeit ist, daß der Typ der Schreibaufgabe den Textproduktionsprozeß steuert. Sie untersucht Texte von geübten Schreibern und erhebt Pausen ab einer Länge von einer zehntel Sekunde (der Schreibvorgang der Probanden bzw. Pausen wurde aufgrund von Videoaufzeichnungen bestimmt; die Fallstudien sollten Muster der Körpersprache in Zusammenhang mit Schreibpausen gewinnen;). Sie zeigt u.a., daß Pausen innerhalb von Erörterungen und innerhalb von Texten, die einen Leser explizit beeinflussen sollen, länger sind als Pausen in Berichten. Sie zeigt auch, daß die längsten Pausen an Satzgrenzen auftreten. Auch andere Forscher zeigen, daß lange Pausen am häufigsten zwischen Sinneinheiten (Absätzen) signifikant sind.

Vor diesem Hintergrund formulieren Flower \& Hayes (1981) zwei Hypothesen: eine linguistische Hypothese, nach der Schreiber pausieren, um zu planen, was als nächstes zu schreiben ist, und eine sog. rhetorische bzw. kognitive Hypothese, nach der längere Pausen mit Bedürfnissen globaler Planung in Verbindung stehen. Für die linguistische Hypothese sprechen Ergebnisse von Matsuhashi (1981, 1982): Pausen treten bevorzugt bei höherer gedanklicher Komplexität auf, ferner an Satzgrenzen. Andererseits scheinen die Ergebnisse in einem Zusammenhang mit der Entwicklung der Schreibfähigkeit und mit der Textsorte (Bericht und Erzählung folgen dem Leitfaden des zeitlichen Nacheinander) zu stehen. Für die rhetorische bzw. kognitive Hypothese spricht, daß sie dem für ausgebildete Schreiber (Bereiter \& Scardamalia 1983) charakteristischen Planen auf mehreren Ebenen gerecht wird und zugleich die satzbezogene Planung einzubeziehen vermag.

Im einzelnen gehen Flower \& Hayes (1981) so vor, daß sie die verbalen Protokolle lauten Denkens in "composing episodes" zerlegen; das sind Einheiten, in denen sich der Schreiber auf ein und denselben Fokus konzentriert. Sie nehmen an, daß die Grenzen zwischen solchen Episoden, in denen sich ein Fokuswechsel vollzieht, eine maßgebliche Quelle für sog. prägnante Pausen sind (Pausen als Indikatoren).

Auch anderen Forschern ist die mögliche Schlüsselrolle der Schreibpausen nicht verborgen geblieben: De Beaugrande (1984) beschäftigt sich vor dem Hintergrund seines Modells, nach dem sich Textproduzieren ständig parallel auf mehreren Ebenen vollzieht, mit den Schreibpausen; Chafe (1977), Sprechen und Schreiben kontrastierend, beschäftigt sich auch mit Schreibpausen.

Die Beobachtung der Pausen als Indikatoren kognitiver Prozesse beim Schreiben eröffnet zweifellos einen Zugang zum Textproduktionsprozeß. Auffallend ist zunächst einmal der große Anteil der Pausenzeit an der Gesamtproduktionszeit. Die Gesamtzeit wird von Schreibern jeweils zur Hälfte für Schreiben und für Pausen aufgewendet, die Sprechzeit aber zu 2/3 für Sprechen und 1/3 für Pausen. Das zeigte nur ein Vergleich (s. Eigler et al. 1990: 236). Laut anderer Ergebnisse sind die Unterschiede noch viel größer. Das Verhältnis der Pausenzeiten zwischen Aussagenketten ist bei Sprechern und 
Schreibern 1:10, zwischen und innerhalb von Aussagen sogar 1:16 (vgl. Eigler et al. ebenda).

Um den Anteil der Pausenzeit an der gesamten Produktionszeit angemessen würdigen zu können, müßte man wissen, wie viel Zeit zur Produktion vergleichbarer mündlicher Texte benötigt wird und wie groß in diesem Fall der Anteil der Pausenzeit ist.

Alle empirischen Studien weisen darauf hin, daß Pausen ein integraler und notwendiger Bestandteil des Produktionsprozesses sind. Auftretenwahrscheinlichkeiten und variierende Längen von Pausen an spezifischen Textstellen lassen Rückschlüsse auf den Beginn und die relative Wichtigkeit eines mit dieser Textstelle korrelierenden Planungsprozesses zu.

Innerhalb der mündlichen Sprachproduktionsforschung gab es schon viel früher empirische Untersuchungen (z.B. Goldman-Eisler 1958, Maclay \& Osgood 1959 u.a.). Maclay \& Osgood (1959) nahmen eine Differenzierung verschiedener hesitation pauses vor: repeats, worunter sie Wiederholungen von Äußerungen verstehen, false starts, d.h. Selbstkorrekturen unvollständiger Äußerungen, filled pauses als Pausen, in denen Haesitationssignale wie "äh" oder "hm" gesprochen werden, und unfilled pauses, in denen zwischen der Produktion zweier Wörter eine wahrnehmbare "leere" Pause liegt. Des weiteren zeigten sie, daß die meisten Pausen vor Inhaltswörtern liegen und wenigere vor Funktionswörtern, während andere Forscher zeigten, daß Pausen hauptsächlich an grammatischen. Verbindungsstellen auftreten (vgl. Henderson, Goldman-Eisler \& Skarbek 1966).

Nach Butterworth (1975) korreliert zwar der rhythmische Wechsel von Pausen und flüssigem Sprechen mit syntaktischen Einheiten, syntaktische Prozesse sind jedoch nicht die Ursache für diesen Wechsel. Ursache sind semantische ideas, die in Sprache transformiert werden müssen (Butterworth 1975: 76). Syntaktische Planungsprozesse sind somit semantischen nachgeordnet.

Aufgrund vieler empirischer-Untersuchungen läßt sich zusammenfassend sagen, daß die Auftretenswahrscheinlichkeit von Pausen am Satzanfang größer ist als an anderen Positionen im Satz (vgl. z.B. Holmes 1984, 1988; Butterworth 1980).

\section{FAZIT}

In den vorangegangenen Abschnitten wurden die derzeit bekanntesten kognitiven Modelle des schriftlichen Textproduzierens dargestellt. Diese Modelle sind stark an der Gedächtnisforschung orientiert, was dazu führt, den Textproduktionsprozeß als Interaktion von individuellem Gedächtnis und Text zu modellieren. Jedes der vier beschriebenen Modelle eröffnet einen Zugang zu Wechselbeziehungen von Wissen und Textproduzieren. Dabei wird das Wissen nicht nur aktiviert, um dann niedergeschrieben 
zu werden, sondern es wird aktualisiert, d.h. in einen Zusammenhang gebracht, der dem Thema, Zweck und Leser des Textes entspricht. Somit ist Textproduzieren eine zielgerichtete und partnerbezogene Handlung, bei der rekursiv Phasen der Planung, des Übersetzens und des Überarbeitens wiederholt durchlaufen werden.

Allen diesen Modellen sind folgende Parameter gemeinsam:

- alle Modelle sind dynamische Prozeßmodelle;

- Prozesse arbeiten parallel und rekursiv, d.h.

- es werden mehrere Verarbeitungsebenen angenommen;

- $\quad$ eine weitere Differenzierung des Textproduktionsprozesses geschieht in drei Schritten:

1. "Problem" (bzw. Problemlösen)

2. "Rapräsentation"

3. "Makrostruktur"

Beim Textproduzieren, als Problemlösen verstanden (z.B. Bereiter 1980, de Beaugrande 1984, Scardamalia \& Bereiter 1986), werden alle anderen Textproduktionsprozesse wie z.B. das assoziative Schreiben, nicht als Problemlöseprozesse angesehen, also auch nicht die Formen alltäglichen Schreibens, sofern sie auf sachlicher Ebene kein Problem bieten und das Textproduzieren selbst durch mehr oder weniger feste Textschemata gesteuert wird und insofern keine Probleme aufwirft. Auch das Textproduzieren als epistemischer Effekt wird eng mit dem Textproduzieren als Problemlösen verknüpft (vgl. z.B. Chafe (1977), der einen spezifischen epistemischen Effekt des Textproduzierens annimmt).

In den meisten Modellen ist die generierte mentale Repräsentation eine propositionale Prädikat-Argument-Struktur. Es wird angenommen, daß mit dem Stellen eines Themas Wissen aktualisiert wird, d.h. sich eine erste Repräsentation des Sachverhalts bildet. Es wurde nachgewiesen, daß die Texte, die unter der Bedingung "Themenbereich bearbeitet" geschrieben wurden, nicht nur insgesamt länger sind und mehr kohärente Äußerungen enthalten als die Texte, die unter der Bedingung "Themenbereich nicht bearbeitet" geschrieben wurden (vgl. z.B. McCutchen 1986), sondern im Durchschnitt auch eine größere maximale Tiefe aufweisen. Der größere Textumfang ergibt sich also zum einen dadurch, daß mehr Inhalte präsentiert werden (horizontale Textentwicklung, d.h. mehr Aussagen in nebengeordneter Position), zum anderen dadurch, daß Inhalte differenzierter dargestellt werden (vertikale Texentwicklung, d.h. mehr Aussagen in über- bzw. untergeordneter Position).

Des weiteren weisen die Untersuchungen darauf hin, daß Gruppen von Versuchspersonen mit unzulänglichem Wissen in bezug auf ein Thema keine gedankliche Repräsentation hervorbringen können, sondern die Themenstellung wörtlich aufnehmen und - wenn es überhaupt zum Produzieren eines Textes kommt sich in der Art eines "knowledge telling" auf die einzelnen Begriffe der Themenstellung 
beziehen, oder aber sie verschieben die Themenstellung, um so handlungsfähig zu werden.

Der Begriff "Makrostruktur" stammt schon aus van Dijks Dissertation (s. van Dijk 1972 und van Dijk \& Kintsch 1983), wo sie vielmehr für Produktion konzipiert war. In der Textverarbeitungsforschung wird mit "Makrostruktur" die Wissensstruktur gemeint, die sich beim Verarbeiten eines Textes über dessen Mikrostruktur aufbaut, d.h. was der Text für den Leser bedeutet. Der Textproduzent weiß bei Abschluß der Arbeit den niedergeschriebenen Text nicht wörtlich, wohl hat sich bei ihm während des Textproduzierens eine Makrostruktur aufgebaut, vergleichbar der Makrostrukturbildung beim Textverarbeiten. Im Zuge der "schematization"-, "framing"- und "categorization"Prozesse bildet sich eine mehr oder wenig reich differenzierte Makrostruktur aus, die anders ist als die anfängliche Repräsentation.

\section{LITERATUR}

Beaugrande, R. de (1984): Text production: Toward a science of composition. Norwood: Ablex.

Beaugrande, R. de \& Dressler, W.U. (1981): Einführung in die Textlinguistik. Tübingen: Niemeyer.

Bereiter, C. (1980): Development in writing. In: L.W. Gregg \& E.R. Steinberg (Eds.): Cognitive processes in writing. Hillsdale: Erlbaum, 73-93.

Bereiter, C. \& Scardamalia, M. (1983): Does learning to write have to be so difficult? In: A. Freedman, I. Pringle \& J. Yalden (Eds.): Learning to write: First language, second language. London: Longman, 20-33.

Bereiter, C. \& Scardamalia, M. (1985): Wissen-Wiedergeben als ein Modell für das Schreiben von Instruktionen durch ungeübte Schreiber. Unterrichtswissenschaft 4, 319-333.

Bereiter, C. \& Scardamalia, M. (1987): The psychology of written composition. Hillsdale: Erlbaum.

Borkowski, J.G. \& Krause, A.J. (1985): Metacognition and attributional beliefs. In: D'Ydewalle, G. (Ed.). Cognition, information, and motivation. Amsterdam: Elsevier, 557-567.

Bresnan, J. (Ed.): The mental representation of grammatical relations. Cambridge, MA: MIT Press.

Bruner, J.B. \& Olson, J.D. (1976): Symbole und Texte als Werkzeuge des Denkens. In: G.C. Steiner (Ed.): Die Psychologie des 20. Jahrhunderts. Bd. 7: Piaget und die Folgen. München: Kindler, 306-320.

Butterworth, B. (1975): Hesitation and semantic planning in speech. Journal of Psycholinguistic Research 4, 75-87.

Butterworth, B. (1980): Evidence from pauses in speech. In: B. Butterworth (Ed.): Language production. Vol. 1: Speech and talk, 155-176. London: Academic Press. 
Chafe, W.L. (1977): Creativity in verbalization and its implications for the nature of stored knowledge. In: R.O. Fredle (Ed.): Discourse production and comprehension. Norwood: Ablex, 41-55.

Chafe, W.L. (1985): Linguistic differences produced by differences between speaking and writing. In: D.R. Olson, N. Torrance \& A. Hildyard (Eds.): Literacy, language, and learning. Cambridge: Cambridge University Press, 105-123.

Cooper, C. \& Matsuhashi, A. (1983): A theory of the writing process. In: M. Martlew (Ed.): The psychology of written language. Chichester: Wiley, 3-39.

Dijk, T.A. van (1972): Some Aspects of Text Grammar. Den Haag: Mouton.

Dijk, T.A. van (1980): Textwissenschaft. Eine interdisziplinäre Einführung. München: dtv.

Dijk, T. van \& Kintsch, W. (1978): Cognitive psychology and discourse: Recalling and summarizing stories. In: W.V. Dester (Ed.): Current trends in textlinguistics. Berlin: de Gruyter, 61-80.

Dijk, T. van \& Kintsch, W. (1983): Strategies of discourse comprehension. New York: Academic Press.

Dressler, W.U. (1989): Semiotische Parameter eìner textlinguistischen Natürlichkeitstheorie. Wien: Österreichische Akademie der Wissenschaften.

Ehlich, K. (1980): Der Alltag des Erzählens. In: K. Ehlich (Ed.): Erzählen im Alltag. Frankfurt / M.: Lang, 11-27.

Ehlich, K. \& Rehbein, J. (1979): Sprachliche Handlungsmuster. In: H.-G. Soeffner (Ed.): Interpretative Verfahren in den Sozial- und Textwissenschaften. Stuttgart, 243-274.

Eigler, G., Jechle, T., Merziger, G. \& Winter, A. (1990): Wissen und Textproduzieren. Tübinger: Narr.

Fischer, P.M. \& Mandl, H. (1980): Selbstwahrnehmung und Selbstbewertung beim Lernen. Tübingen: DIFF-Forschungsbericht Nr. 10.

Flower, S.L. \& Hayes, J.R. (1977): Problem-solving strategies and the writing process. College English 39, 449-461.

Flower, S.L. \& Hayes, J.R. (1980): The dynamics of composing: Making plans and juggling constraints. In: L.W. Gregg \& Hillsdale: Erlbaum, 31-50.

Flower, S.L. \& Hayes, J.R. (1981a): A cognitive process theory of writing. College Composition and Communication 32, 365-387.

Flower, S.L. \& Hayes, J.R. (1981b): The pregnant pause: Aninquiry into the nature of planning. Researche in the Teaching of English 15, 229-243.

Flower, S.L. \& Hayes, J.R. (1984): Images, plans, and prose. The representation of meaning in writing. Written Communication 1, 120-160.

Fromkin, V.A. (Ed.) (1973): Speech errors as linguistic evidence. Den Haag: Mouton.

Fromkin, V.A. (1988): Grammatical aspects of speech errors. In: F.J. Newmeyer (Ed.): Linguistics: The Cambridge survey. Vol. II: Linguistic theory: Extensions and implications. Cambridge: University Press, 117-138.

Goldman-Eisler, F. (1951): The measurement of time sequences in conversational behaviour. British Journal of Psychology 42, 355-362. 
Goldman-Eisler, F. (1954): On the variability of the speed of talking and on its relation to the length of utterrances in conversations. British Journal of Psychology 45, 94-107.

Goldman-Eisler, F. (1958): Speech production and the predictability of words in context. The Quarterly Journal of Experimental Psychology 10, 96-106.

Hayes, D.P. (1988): Speaking and writing: Distinct patterns of word choice. Journal of Memory and Language 27. 572-585.

Hayes, D.P. \& Flower, L.S. (1980): Identifying the organization of writing processes. In: L.W. Gregg \& E.R. Steinberg (Eds.): Cognitive processes in writing. Hillsdale: Erlbaum, 3-30.

Henderson, A., Goldman-Eisler, F. \& Skarbek, A. (1966): Sequential temporal patterns in spontaneous speech. Language and Speech 9, 207-216.

Herrmann, T. (1985): Allgemeine Sprachpsychologie. Grundlagen und Probleme. München: Urban und Schwarzenberg.

Holmes, V.M. (1984): Sentence planning in a story task. Language and Speech 27, 115-134.

Holmes , V.M. (1988): Hesitations and sentence planning. Language and Cognitive Processes 3, 323-361:

Karmiloff-Smith, A. (1985): Language and cognitive processes from a developmental perspective. Language and Cognitive Processes 1, 61-85.

Kintsch, W. \& Dijk, T. van (1978): Toward a model of text comprehension and production. Psychological Review 85, 363-394.

Kluwe, R. (1979): Wissen und Denken. Stuttgart: Kohlhammer.

Kluwe, R. (1981): Metakognition. In: W. Michaelis (Ed.): Bericht über den 32. Kongreß der Deutschen Gesellschaft für Psychologie in Zürich 1980. Göttingen: Hogrefe, 46-258.

Ludwig, O. (1983): Einige Gedanken zu einer Theorie des Schreibens. In: S. Grosse (Ed.): Schriftsprachlichkeit. Düsseldorf: Schwann, 37-73.

Luria, A.R. (1973): The working brain. New York: Penguin Books.

Maclay, H. \& Osgood, C.E. (1959): Hesitation phenomena in spontaneous English speech. Word 15, 19-44.

Makovec-Černe, J. (1991): Die Thema-Rhema-Gliederung in deutschen und slowenischen Texten. Frankfurt/M.: Lang.

Makovec-Černe, J. (1992): Die thematische Organisation von Texten. Eine kontrastive Untersuchung. Folia Linguistica XXVI, 3-4, 435-452.

Matsuhashi, A. (1981): Pausing and planning. The tempo of written discourse production. Research in the Teaching of English 15, 113-134.

Matsuhashi, A. (1982): Explorations in the real-time production of written discourse. In: M. Nystrand (Ed.): What writers know: The language, process, and structure of written discourse. New York: Academic Press, 269-290.

McCutchen, D. (1986): Domain knowledge and linguistic knowledge in the development of writing ability. Journal of Memory and Language 25, 431-444. 
McCutchen, D. \& Perfetti, C.A. (1982): Coherence and connectedness in the development of discourse production. Text 2, 113-139.

Meringer, R. \& Meyer, K. (1895): Versprechen und Verlesen: Eine psychologischlinguistische Studie. Stuttgart: Göschen'sche Verlagsbuchhandlung.

Molitor, S. (1984): Kognitive Prozesse beim Schreiben. Tübingen: Deutsches Institut für Fernstudien, Arbeitsbericht Nr. 31.

Norman, D.A. (Ed.) (1981): Perspectives on cognitive science. Norwood: Ablex.

Pascual-Leone, J. \& Smith, J. (1969): The encoding and decoding of symbols by children: A new experimental paradigm and a neo-Piagetian model. Journal of Experimental Child Psycology 8, 328-355.

Rehbein, J. (1977): Komplexes Handeln. Elemente zur Handlungstheorie der Sprache. Stuttgart.

Scardamalia, M., Bereiter, C. \& Steinbach, R. (1984): Teachability of reflective processes in written composition. Cognitive Science 8, 173-190.

Scardamalia, M. \& Bereiter, C. (1986): Research on written composition. In: M.C. Wittrock (Ed.): Handbook of research on teaching. New York: Macmillan Education Ltd, 778-803.

Scardamalia, M. \& Bereiter, C. (1987): Knowledge telling and knowledge transforming in written composition. In: S. Rosenberg (Ed.): Advances in applied psycholinguistics. Vol. 2: reading, writing, and language learning. Cambridge: University Press, 142-175.

Shechter, T.M. \& Toglia M.P. (1985): New directions in cognitive science. Norwood: Ablex.

Stallard, C.K. (1974): An analysis of the writing behavior of good student writers. Research in the Teaching of English 8, 206-218.

Tannen, D. (1985): Relative focus on involvement in oral and written discourse. In: D.R. Olson et al. (Eds.): Literacy, Language and Learning. Cambridge: University Press, 124-147.

Voss, J.F. et al. (1980): Text generation and recall by high-knowledge and low-knowledge individuals. Journal of Verbal Learning and Verbal Behavior 19, 651-667.

Watzlawick, P. et al. (1974): Menschliche Kommunikation. Formen, Störungen, Paradoxien. Bern: Lang.

Winter, A. (1992): Metakognition beim Textproduzieren. Tübingen: Narr.

Witte, S.P. \& Faigley, L. (1981): Coherence, cohesion, and writing quality. College Composition and Communication 32, 189-204.

Wygotski, L.S. (1977): Denken und Sprechen (1934). Frankfurt/M.: Fischer. 


\section{Povzetek \\ BESEDILNA PRODUKCIJA \\ KOGNITIVNI MODELI}

Eno izmed področij kognitivne lingvistike je tudi produkcija pisnih in ustnih besedil. Medtem ko so prve sistematične kognitivne raziskave ustnih besedil stekle že v zgodnjih petdesetih letih (predvsem v ZDA), pa so se sistematične kognitivne raziskave produkcije pisnih besedil začele šele $v$ osemdesetih letih ( $v$ ZDA, kmalu zatem pa tudi v Evropi, zlasti $v$ Nemčiji). Značilnost teh raziskav je, da niso usmerjene $v$ besedilo kot produkt, temveč proučujejo sam proces nastajanja besedila. Kognitivna teorija besedilne produkcije naj bi torej opisala ter pojasnila, kako se mentalna reprezentacija našega (deklarativnega, proceduralnega, epizodičnega) znanja "prevede" v jezikovne strukture.

$\mathrm{V}$ prispevku sem najprej orisala splošne in obenem temeljne kognitivne procese $\mathrm{v}$ teku nastajanja besedila (2. poglavje), nato pa opisala trenutno najbolj (pri)znane kognitivne modele pisne besedilne produkcije. To so: Hayes \& Flowerjev model, de Beaugrandov model, Bereiterjev oz. Bereiter \& Scardamaliajev model in Cooper \& Matsuhashijev model (3. poglavje). Vzporedno z modeli so predstavljene glavne empirične metode, na rezultatih katerih temeljijo teoretične predpostavke posameznih modelov (4. poglavje).

Vsak izmed modelov na svoj način omogoča vpogled $v$ interakcijo odnosov med védenjem in besedilno produkcijo. Vsem modelom pa so skupni naslednji parametri: (1) vsi modeli so dinamični in procesno usmerjeni, (2) procesi delujejo paralelno in rekurzivno, (3) prva groba diferenciacija besedilne produkcije obsega tri stopnje: problem (ustvarjanje/pisanje besedila v smislu reševanja nekega problema), reprezentacija in makrostruktura besedila. 